\title{
Transitando desde la observación a la acción pedagógica en la práctica inicial: Aprender a enseñar con foco en el aprendizaje del alumnado*
}

\author{
Moving from observation to pedagogical actions in initial field experiences: \\ learning to teach with a focus on students' learning \\ Mónica Córtez $z^{a}$ Carmen Montecinos ${ }^{b}$ \\ ${ }^{a}$ P. Universidad Católica de Valparaíso. Correo electrónico: monica.cortez@pucv.cl \\ ${ }^{\text {b }}$ P. Universidad Católica de Valparaíso. Correo electrónico: carmen.montecinos@puvc.cl
}

\begin{abstract}
RESUMEN
Este estudio examina la trayectoria de aprendizaje profesional de seis estudiantes de pedagogía participantes en un programa de innovación en la práctica inicial. La innovación se sustentó en el enfoque sociocultural de aprendizaje. Se realizó una versión abreviada de investigación evolutiva en el trabajo (Engeström) en los talleres de práctica semanal donde ellos participaron junto con tutores universitarios, profesora mentora e investigadora Notas de campo y análisis de las conversaciones evidenciaron cómo la incorporación de tareas auténticas a la labor docente y herramientas de mediación con voces de la academia y del centro escolar, movilizaron a los practicantes desde un foco en su enseñanza hacia un foco en el aprendizaje de sus estudiantes. Estos resultados ejemplifican la importancia de construir nuevas configuraciones para el currículo de la práctica inicial que enfrenten a candidatos a tareas auténticas al quehacer docente, con mediación conjunta de pares, profesores mentores y tutores, que fortalezcan su agencia profesional.

Palabras claves: Formación inicial docente, formación práctica, práctica inicial, enfoques socioculturales aprendizaje docente
\end{abstract}

\begin{abstract}
The current study examines the professional learning trajectory of six teacher candidates in their first school placement. They were participants in a redesigned practicum component guided by sociocultural learning theory. Data were collected through the use of an abbreviated form of the Development Work Research (Engeström) during the weekly meeting of candidates, cooperating teachers and university practicum supervisor. An analysis of field notes and transcripts of the conversations among participants evidences how performing tasks authentic to teaching and the mediating tools used by cooperating teachers and supervisors moved candidates from a focus on their teaching to a concern for how much their students were learning. These findings exemplify the importance of designing and implementing the initial practicum course as a space that enables teacher candidates to work on authentic teaching tasks, with the joint mediation of peers, cooperating teacher and university tutor, to strengthen professional agency.
\end{abstract}

Key words: Initial teacher preparation, practicum, initial practicum, sociocultural approach to teacher learning

Este estudio fue financiado por Fondecyt (Proyecto $\mathrm{N}^{\circ} 1110505$ ). Financiamiento adicional fue provisto para la primera autora a través de CONICYT-FB 003. Este artículo presenta resultados parciales de la tesis doctoral de la primera autora bajo la dirección de la segunda autora. 
Estudios Pedagógicos XLII, N 4: 49-68, 2016

TRANSITANDO DESDE LA OBSERVACIÓN A LA ACCIÓN PEDAGÓGICA EN LA PRÁCTICA INICIAL: APRENDER A ENSEÑAR CON FOCO EN EL APRENDIZAJE DEL ALUMNADO

\section{INTRODUCCIÓN}

Las experiencias de formación práctica que realizan los futuros profesores en centros escolares han sido reconocidas como uno de los componentes más influyentes en la formación inicial del profesorado (Clift \& Brady, 2005; Musset, 2010; Ortúzar, Flores, Milesi \& Cox, 2009; Zeichner, 2010). En Chile, desde fines de los noventa, la política educativa ha prestado especial atención a la formación práctica y las condiciones requeridas para su adecuado desarrollo (Ávalos, 2002; Comisión sobre Formación Inicial de Docentes, 2005; Panel de Expertos, 2010). Existe consenso en la necesidad de que futuros profesores establezcan contacto temprano con los centros escolares y que su proceso de formación profesional contemple un diseño progresivo de complejidad creciente (Ávalos, 2002). El aumento de formación práctica por sí misma no asegura una mejora en la formación del profesorado (Lunenberg \& Korthagen, 2009). De hecho, los futuros profesores reconocen su importancia, pero señalan que no siempre estas experiencias resultan útiles para su proceso formativo (Ortúzar et al., 2009).

Generar una formación práctica en la intersección del saber escolar y del saber universitario involucra: (a) la construcción de una cultura de colaboración universidadcentros escolares (Montecinos, Walker, y Maldonado, 2014; Zeichner, Payne \& Brayko, 2012), (b) comprensiones teóricas respecto del aprendizaje del profesor en formación situado en un centro escolar (Putnam \& Borko, 2000) y (c) el diseño conjunto con el centro escolar de un currículo de formación práctica que contribuya al mejoramiento simultáneo de la formación inicial y de las oportunidades de aprendizaje de los escolares. La investigación nacional e internacional orientada por enfoques socioculturales del proceso de aprender a enseñar sugieren que el currículo de formación práctica tiene mayor impacto cuando los profesores en formación: (a) ponen en el centro de su quehacer profesional el aprendizaje de sus estudiantes (Korthagen, Loughran \& Russell, 2006) y (b) trabajan con un alumnado diverso, asumiendo la responsabilidad para que todos sus estudiantes tengan oportunidades de aprender (Darling-Hammond, 2013; Ortúzar et al., 2009).

La participación de los futuros profesores en los centros escolares es una instancia para aprender a: (a) desarrollar y actualizar competencias; (b) integrar conocimientos y experticias; (c) desarrollar saberes propios al mundo de la práctica (Correa Molina, 2014); (d) participar en complejos procesos de interacción social con alumnos y profesionales para confrontar y transformar sus creencias (Méndez Zaballos, 2012); y (e) desarrollar su juicio profesional, al problematizar, indagar e idear soluciones para mejorar sus prácticas pedagógicas (Ávalos, 2002). El fin último de la formación inicial de profesores es el desarrollo de la agencia profesional, experticia colectiva y transformativa requerida para atender la alta complejidad de los contextos educativos (Engeström, 2004; Edwards, 2011). Esta agencia se relaciona con la capacidad para tomar decisiones e involucrarse en acciones orientadas a la consecución de metas.

El presente estudio examina una experiencia que modificó la práctica inicial centrada en tareas de observación del quehacer del aula escolar hacia tareas en las cuales los practicantes asumen la enseñanza de los estudiantes. Esta innovación a la práctica inicial fue diseñada en concordancia con los principios ya reseñados: co-diseño entre los tutores de la universidad y la docente mentora del aula escolar, desempeño de tareas auténticas a través de las cuales los practicantes asumieron gradualmente responsabilidades por el aprendizaje de los estudiantes, trabajo colaborativo entre practicantes, y procesos de 
mediación integrando las voces de la profesora del aula, de dos tutores de la universidad y de seis practicantes. Utilizando el diseño de estudio de caso, durante un semestre se registraron las conversaciones en la reunión semanal del taller de práctica. Los resultados del análisis de las conversaciones entre los miembros del taller se reportan para ilustrar la trayectoria de aprendizaje profesional de los seis profesores en formación en su práctica inicial.

\section{ANTECEDENTES TEÓRICOS Y EMPÍRICOS}

\subsection{FORMACIÓN PRÁCTICA INICIAL: TENSIONES Y POSIBILIDADES}

La primera experiencia práctica en el centro escolar marca un hito en la trayectoria formativa de futuros profesores (Correa Molina, 2014). Estas experiencias tienden a desencadenar en los candidatos determinadas Gestalts (formas de percibir e interpretar) que pueden obstaculizar el proceso de aprender a enseñar (Borko, 2004). En Chile, la práctica inicial se realiza en el primer o segundo año de la carrera y comúnmente conlleva actividades de observación e indagación centradas en los estudiantes, en la institución escolar y en la construcción de su identidad profesional (Montecinos et al., 2011). Contreras, Rittershaussen, Montecinos, Solís, Núñez y Walker (2010) reportan que el 82\% de los profesores en formación que cursaron esta práctica señalan la observación como la principal tarea a la que estuvieron abocados mientras estaban en el centro escolar. Esta práctica se acompaña, la mayoría de las veces, de un taller realizado en la universidad donde los practicantes, acompañados por un profesor universitario, analizan y reflexionan estas experiencias (Ávalos, 2002; Solís, Núñez, Contreras, Rittershaussen, Montecinos \& Walker, 2011).

Un estudio realizado en Chile muestra que al momento de identificar barreras y obstáculos en la formación práctica de futuros profesores, los docentes directivos de los centros escolares resultaron especialmente críticos de la práctica inicial (Montecinos, Cortez \& Walker, 2015). Dentro de las críticas señalaron: (a) visitas cortas y esporádicas de los profesores en formación, (b) estar centradas casi exclusivamente en tareas de observación, y (c) escasa presencia y apoyo de tutores universitarios. Estos aspectos resultaban en una carga adicional para el centro escolar, contrario al apoyo que buscaban al aceptar recibir practicantes. Estos docentes directivos sugieren que las experiencias prácticas se focalicen desde el comienzo en tareas de apoyo hacia alumnos y mentores, situando al practicante desde un inicio más como docente que como estudiante universitario (Montecinos et al., 2015). Estas opiniones son coincidentes con enfoques socioculturales que plantean que será a través de acciones e interacciones en el aula, con estudiantes y profesores, que futuros profesores aprenderán a interpretar los acontecimientos que suceden en el aula y a tomar decisiones que les permitan reconocer y responder a las demandas profesionales (Edwards \& Protheroe, 2003). El presente estudio aborda una innovación en la práctica inicial orientada por el enfoque sociocultural de aprendizaje situado que aborda las críticas que provienen desde el sistema escolar.

\subsection{ENFOQUES SOCIOCULTURALES PARA ENRIQUECER LA PRÁCTICA INICIAL}

Los enfoques socioculturales ofrecen marcos teóricos y metodológicos para comprender cómo los futuros profesores logran desarrollar aprendizaje profesional participando en 
actividades socialmente organizadas (Ludvigsen, Lund, Rasmussen \& Säljö, 2011; OrlandBarak, 2005; Putnam \& Borko, 2000). Nuevas condiciones para aprender emergen a partir de la intersección de tareas en las cuales participan los profesores en su formación inicial, acciones que realizan, herramientas que utilizan y aspectos institucionales-contextuales en los cuales están embebidas sus experiencias prácticas (Ludvigsen et al., 2011).

Los enfoques socioculturales reconocen que el conocimiento requerido para formar a un profesor se encuentra distribuido entre personas, entornos sociales y simbólicos, objetos y artefactos; de ahí la importancia de reconocer, legitimar y hacer confluir las diversas perspectivas de los participantes en la formación práctica (Greeno, 1998). Para estos enfoques, el aprendizaje profesional será fruto de la tensión dinámica que se produce entre las demandas que se realizan desde la práctica y el despliegue de la agencia de futuros profesores mientras aprenden a enseñar en los centros escolares (Hedegaard, 2012). Adecuadas experiencias prácticas permitirán que los profesores en formación vayan conectando los conceptos cotidianos que están aprendiendo en las escuelas con formas de pensamiento más robustas basadas en herramientas conceptuales/teóricas, enriqueciendo así sus conocimientos y decisiones profesionales (Edwards \& Protheroe, 2003).

Korthagen et al. (2006) señalan que se requiere establecer instancias de reflexión estructuradas para que los practicantes compartan experiencias y preocupaciones que emergen. El estudio clásico de Fuller (1969) da cuenta cómo un taller de asesoramiento durante la práctica final posibilitó que candidatos avanzaran desde preocupaciones centradas en sí mismos (propia seguridad, dominio de la materia, opinión de profesores sobre su desempeño, etc.) hacia preocupaciones centradas en sus alumnos (aprendizaje, progreso o avance, estrategias de apoyo, etc.). Para Fuller, la hipótesis explicativa más sustantiva de dicha evolución estuvo en las sesiones de asesoramiento semanal, donde conversaban y reflexionaban junto a sus pares y otros actores educativos.

La creación de configuraciones sociales, donde participan simultáneamente actores educativos de la universidad y del centro escolar, resultan especialmente enriquecedoras, pues en ellas confluyen propósitos, experticias, conocimientos, emociones, propiciando la construcción de conocimiento compartido (Edwards, 2011). Las instancias de trabajo colaborativo con sus pares y con profesores con mayor experticia se vuelven sitios para ampliar la comprensión mutua respecto a tareas y problemas comunes. A partir de las interacciones con pares y con otros actores educativos el futuro profesor irá reflexionando respecto a su quehacer e irá transformando sus conceptualizaciones respecto a la enseñanza, el aprendizaje y a la relación entre ambos procesos (Blanton, Berenson \& Norwood, 2001). Los actores educativos que participan de estas instancias logran asî ampliar su propio sentido de quiénes son y hacia dónde van como profesionales (Edwards $\&$ Mutton, 2007).

\section{DESCRIPCIÓN DE LA INNOVACIÓN IMPLEMENTADA}

\subsection{MODALIDADES DE TRABAJO EN AULA}

El rediseño de la práctica inicial tuvo como principal propósito que, en esta instancia, los futuros profesores desarrollaran una comprensión profunda respecto de cómo aprenden los estudiantes. Para lograr este propósito, los tutores junto a la docente de aula, la 
mentora, diseñaron tres modalidades de trabajo secuenciales y de progresión creciente en complejidad. Los practicantes transitaron desde la modalidad de ayudantía en aula (primeras 5 semanas) apoyando a la mentora, hacia tutoría a grupos pequeños y estables en función a la planificación de la clase que entregaba la mentora (durante 6 semanas), para finalizar diseñando y realizando docencia colegiada al grupo curso (últimas 6 semanas).

La primera modalidad, ayudantía, demandó un foco en el curso completo que resultó complejo debido a la escasa comprensión que los practicantes tenían acerca de su rol en el aula. Los practicantes parecían encontrarse inmersos en una experiencia que, si bien habían vivenciado desde el rol de estudiantes, por primera vez la experimentaban desde la posición de profesores en formación.

La segunda modalidad, apoyo tutorial, era la tarea central de la práctica inicial diseñada desde la universidad. Su foco de actuación involucró apoyar el aprendizaje de un grupo de 5-6 alumnos. En esta modalidad, la mentora planificaba la clase y los practicantes implementaban la planificación con su grupo. Esto otorgó estructura y sentido a su quehacer. A medida que iban conociendo a sus alumnos y desarrollando apoyo pedagógico, a través de las tutorías iban construyendo su rol de profesores en formación y aportando ideas en la planificación de clases que proponía la mentora.

La tercera modalidad, diseño e implementación de clases colegiadas y situadas, implicó un foco de actuación con el curso completo. El conocimiento adquirido acerca de las características y necesidades de aprendizaje de los estudiantes, las relaciones de confianza establecida con el curso y todo el bagaje construido a partir del trabajo tutorial, permitió que esta tarea se situará en la zona de desarrollo próximo de estos seis practicantes. Con la mediación brindada en el taller, los practicantes lograron desarrollar las tareas de planificación y enseñanza con claro impacto en los logros de aprendizaje de los escolares a su cargo.

\subsection{HERRAMIENTAS DE MEDIACIÓN: TALLER DE PRÁCTICA POLIFÓNICO}

El taller de práctica semanal fue cambiando su modalidad de trabajo a través de las sesiones, llegando a convertirse en una herramienta de mediación que propició el aprendizaje de los practicantes. Inicialmente, se utilizó un modelo de trabajo con tareas complementarias entre tutores y la mentora; cada uno reuniéndose por separado con los practicantes, tal como se realizaba la práctica inicial antes de la innovación propuesta. Cuando los practicantes fueron asumiendo tareas de tutoría, comenzaron a demandar un trabajo coordinado que les permitiera una buena interacción pedagógica. Estas coordinaciones fueron de carácter instrumental, y si bien permitieron abordar aspectos prácticos en el aula, no posibilitaban aprender de manera conjunta. La tarea de diseñar e implementar clases colegiadas, transformó el taller en un dispositivo de mediación y trabajo colaborativo entre los miembros de la tríada. Para desarrollar esta tarea se requirió de la confluencia de todas las voces participantes en la construcción de un objeto (motivo) compartido: el aprendizaje de los alumnos del centro escolar. En esta confluencia, los profesores en formación aportaron el conocimiento construido respecto a sus alumnos, y la profesora mentora y los tutores contribuyeron con sus saberes y experticias. Utilizando la propuesta de investigación evolutiva en el trabajo (Engeström, 2005), la investigadora aportó la voz de los estudiantes de los cursos en los cuales los practicantes enseñaron. 


\section{MÉTODO}

\subsection{DISEÑO}

Se utilizó un diseño de estudio de caso longitudinal (Serrano, 1998) que permitió examinar en profundidad el trabajo cotidiano realizado por la tríada formativa durante un semestre del desarrollo del taller de práctica. Este estudio de caso se realizó a través de la metodología "investigación evolutiva en el trabajo", propuesta por Engeström (2005).

\subsection{CONTEXTO DE INVESTIGACIÓN}

Esta investigación fue realizada en el marco de una Red de Asociación Universidad - Sistema Escolar en Chile, orientada por los principios de las Escuelas de Desarrollo Profesional (Professional Development School). Estas instancias de trabajo reúnen a profesores en ejercicio, formadores de profesores, estudiantes de pedagogía e investigadores. Los objetivos perseguidos por las Escuelas de Desarrollo Profesional contemplan: mejorar la formación inicial y continua de profesores, mejorar aprendizajes de alumnos del centro escolar, y desarrollar investigación conjunta para mejorar prácticas de enseñanza (DarlingHammond, 1994).

Orientados por los principios y objetivos de este modelo de desarrollo profesional, el año 2014 se conformó esta Red de Asociación en el marco de un Convenio de Desempeño de Formación Inicial Docente (MECESUP). Esta Red, que reunió a seis carreras de pedagogía de una universidad de la región de Valparaíso y a seis centros escolares de la misma región, posibilitó implementar innovaciones en el componente de formación práctica de estas seis carreras. Este estudio recoge la experiencia del taller de práctica de una de estas asociaciones durante el segundo semestre académico del año 2014 mientras se implementaba un programa de innovación en la práctica inicial.

\subsection{PARTICIPANTES}

La asociación estuvo conformada por una carrera de Pedagogía en Educación Media en Castellano y una escuela básica municipal, ambas ubicadas en la Región de Valparaíso. Los seis profesores en formación que se ofrecieron voluntariamente a participar en esta práctica inicial fueron destinados a trabajar en $7^{\circ}$ y $8^{\circ}$ básico, cursos donde la docente mentora enseñaba la asignatura de Comunicación y Lenguaje. Fueron distribuidos tres por curso, contemplando criterio de género al tomar la decisión.

En el taller de práctica semanal participaron: la profesora mentora de 5 años de experiencia y permanencia en dicha escuela, dos tutores universitarios de vasta experiencia docente en centros escolares previo a su trabajo en la universidad, 4 profesoras y 2 profesores en formación, y una de las autoras de este artículo. Lo/as profesores en formación estaban cursando su cuarto semestre de la carrera de pedagogía.

\subsection{PRODUCCIÓN DE DATOS}

Se realizó una versión abreviada de la Investigación Evolutiva en el Trabajo (Engeström, 2005). Se optó por esta metodología, ya que permite estudiar las transformaciones y el 
aprendizaje que se produce mientras se enfrentan problemas auténticos en el desempeño laboral. Se sustenta en el concepto de doble estimulación que tiene sus raíces en la obra de Vygotsky, Luria y Leontiev (Eri, 2013). En el transcurso del trabajo, los participantes enfrentan una situación problemática que va más allá de sus capacidades (primer estímulo) y, para resolverla, utilizan disposiciones (personales y sociales) y herramientas (conceptuales y materiales) disponibles (segundos estímulos). Estos segundos estímulos les permitirán analizar y dar sentido a sus prácticas, a los objetos (motivos) de éstas y a las características de la organización que le dan forma. Es así como el diálogo y debate entre participantes actúan como fuerza impulsora en el desarrollo de nuevos aprendizajes y prácticas (Ellis, 2010).

La investigadora se incorporó a la agenda del trabajo establecida por la tríada formativa. Durante el taller, su rol consistió en potenciar el diálogo y debate entre los participantes. Se utilizaron los siguientes tres tipos de acciones que modelaron una forma de participar en el taller y que podían ser implementadas también por los miembros de la tríada:

- Preguntas de ampliación y profundización, por ejemplo: “(Dirigiéndose a un profesor en formación) Tú mencionaste que ocupaba una estrategia un poco conductista, ¿qué limitaciones le ves a eso y cómo mejorarías tú esa estrategia?"

- Introducir o enriquecer conceptos teóricos para ampliar repertorio interpretativo y de actuación ante situaciones problemáticas, por ejemplo: "un tema a considerar es poder preguntar después de la clase o de varias clases qué aprendieron, no solo en la prueba, monitorear, 'se entendió lo que dije', 'tú lo podrías decir con tus propias palabras'. Eso les va a ir permitiendo visualizar cómo se va construyendo el proceso de aprendizaje en sus alumnos").

- Otorgar evidencias con opiniones de estudiantes del sistema escolar. Dentro de esta última acción se realizó una devolución a mitad del proceso (sesión 5) de evidencias producidas por la investigadora a través de cuestionarios y grupos focales a estudiantes del centro escolar. Por ejemplo, los estudiantes señalaron:

"Porque cuando hay algo que no le entiendo a la profesora muy bien, se lo puedo preguntar a ellos [los practicantes] ya que me ayudan a entender mejor la clase."

"Estoy muy satisfecha ya que he podido aprender y desarrollar más mis conocimientos de la materia, porque con los trabajos grupales todos pueden aportar conocimientos que uno no sabe."

Durante las 17 semanas que estuvieron en el aula, se realizaron 11 sesiones de taller de práctica. En 8 de éstas, se tomaron extensas notas de campo y se realizaron grabaciones de audio, las que fueron transcritas íntegramente (sesiones 1, 2, 4, 5, 6, 7, 8 y 10). El trabajo semanal del taller se efectuó en el centro escolar en reuniones de 2 horas.

\subsection{PROCEDIMIENTO DE ANÁLISIS DE DATOS}

El corpus de datos fue analizado utilizando los principios del análisis sociocultural de discurso. Este contempla la conversación como un modo social de pensar, una actividad intelectual conjunta (Mercer, 2004). A través del discurso un equipo intercambia ideas, toma decisiones y planea acciones. La forma en que conversan revela desde dónde viene y hacia dónde se dirigen; el discurso es una herramienta para cambiar el destino de un equipo (Donnellon, 1996). 
Para el análisis, el corpus de datos se segmentó en función a los tópicos discursivos. Un tópico es una manera intuitiva satisfactoria de describir un principio unificador que distingue un tramo del discurso del siguiente tramo (Brown \& Yule, 1983). El análisis se focalizó en tres aspectos:

1. Los tópicos de discurso (discourse topic), es decir tópicos que convocan la participación de distintos actores. Se procedió así a identificar "sobre qué conversan los participantes" y su evolución en el tiempo.

2. Las preocupaciones (concerns), que refieren a aquellas cuestiones primordiales sobre las cuales profesores en formación tendían a volver en la conversación y cómo estas fueron cambiando (Brown \& Yule, 1983).

3. La construcción de la agencia profesional se refiere a la capacidad de iniciar una acción intencionada que implica voluntad, autonomía, libertad y elección. Se utilizó las categorías de expresiones agenciativas (expressions of agency), propuestas por Haapasaari, Engeström y Kerosuo (2016), y los posicionamientos discursivos que fueron construyendo los practicantes.

Los segmentos codificados fueron luego auditados por la segunda investigadora en un proceso iterativo para llegar a consenso cuando no había acuerdo.

\section{RESULTADOS}

La presentación de los resultados se organiza en tres secciones. En la primera, se analiza e ilustra, con extractos de conversación del taller, cómo los practicantes van transitando desde un foco centrado en la enseñanza hacia un foco centrado en el aprendizaje de los estudiantes del centro escolar. En la segunda, se analiza e ilustra con extractos cómo el aumento de complejidad de las tareas permitió el desarrollo de agencia profesional. En la tercera, se presenta una síntesis que evidencia la relación entre las modalidades de trabajo, los focos de preocupación de los practicantes y las distintas configuraciones de trabajo en el taller de práctica.

\subsection{EL APRENDIZAJE DE LOS ALUMNOS COMO CENTRO DE LA ACTIVIDAD PEDAGÓGICA}

Uno de los aprendizajes clave que fue construido por estos seis profesores en formación fue la transformación de sus creencias respecto al motivo de su quehacer. Se observó cómo los practicantes transitaron desde un foco en aprender a ser buenos enseñantes hacia la creencia que el corazón de su quehacer se encontraba en el aprendizaje de sus alumnos. Este aprendizaje es evidenciado a través de la evolución en los tópicos de discursos y en las preocupaciones que se presentaron en las sesiones de taller.

De los 48 tópicos de discurso identificados a lo largo de las sesiones, 11 pertenecían al tema de la interacción con los alumnos. Este tópico siempre fue incorporado a la conversación por los practicantes. De ahí que se pueda señalar que este tema activó y movilizó la reflexión de los profesores en formación durante todos los talleres. Sin embargo, al avanzar las sesiones y las tareas demandadas, las preocupaciones que surgen en la conversación respecto a este tópico presentaron importantes cambios. 
Durante las semanas que realizaron ayudantía en el aula, apoyando la labor de la mentora, los profesores en formación expresaron sorpresa y nerviosismo al encontrarse con alumnos más interesados en conocerlos que en establecer una relación estrictamente pedagógica. El foco de sus preocupaciones en esta etapa estuvo en sí mismos y su actuación profesional, en cómo posicionarse y validarse en su rol de enseñantes ante los alumnos, en saber cómo reaccionar y actuar ante las interacciones informales y espontáneas planteadas por alumnos. En el siguiente extracto se aprecian estas preocupaciones (sesión 1, semana 1 en aula):

PFT: En particular, trabajé con dos niños que eran muy desordenados y se distraían a cada segundo (...) pero eran ufff al segundo se distraían y peleaban todo el rato entre ellos y como que estábamos hablando de lo que estábamos haciendo y después empezaban a hablar de otra cosa, y me preguntaban "¿que música le gusta a Ud? y yo les decía pucha no pero mejor que trabajemos en esto, no hablemos tanto de música que se yo

T1: No te compliques si te preguntan algo personal, al contrario desarróllalo, qué música, diles la música porque eso te va a permitir crear lazos... en lo educativo lo sustancial es crear lazos, a partir del lazo tu puedes inculcar en el niño ciertas cosas, te das cuenta, y eso muy en buena, así que no te sientas complicada. La profesora nunca te va a decir nada porque tu le dices a una chica que te gusta tal música

PFT: No si no era eso, si no que no me gusta decir tanto lo que a mí me gusta

T2: emm es que tienes que hacerlo

PFT: Igual yo le respondí y le dije cualquier tontera (Todos ríen)

T2: El día de mañana va ser qué equipo de fútbol, dónde viven uds.

PFM: Es que ya nos preguntaron todo eso (ríen todos)

PFT: Sií, de hecho estábamos trabajando y preguntaban cosas solo como para salirse un momento de lo que estaban haciendo, porque de verdad se distraen con mucha facilidad como decía PFM, nos preguntaban que dónde vivíamos y todas esas cosas. De hecho, yo le dije que era del Colo-Colo y él dijo que era de otro y me dijo "ya entonces no hablemos más" (ríen todos)

M: La idea es que exista una relación mutua y de respeto como persona, yo pretendo y espero que así sea, que se logre con ustedes también, porque tampoco es una receta, no puedo decirles anoten chiquillos esto hay que hacer

Tal como se aprecia en el extracto, parece haber un desajuste en la profesora en formación, quien espera que los alumnos asuman rápidamente su rol de "profesora practicante" y que se comporten, desde su rol de alumnos, concentrándose en su trabajo académico. De ahí su desconcierto ante comentarios espontáneos y la curiosidad expresada por los adolescentes hacia su persona. Esta situación, donde alumnos quieren saber finalmente quién es ella, 
produce tensión en la profesora en formación, quien termina utilizando como criterio su preferencia personal de no hablar de sí misma. El consejo de los tutores y mentora, no obstante, no apoya su preferencia y le plantean la necesidad de establecer relaciones de confianza y respeto con los alumnos como base del proceso educativo.

Al transitar hacia la realización de tutorías a pequeños grupos de alumnos, se presentó un primer punto de inflexión en la conversación. Si bien, la interacción con alumnos continúa siendo el tópico predominante en los talleres, el trabajo con un número reducido de alumnos les otorga oportunidades de interacciones más personalizadas y profundas. Sus preocupaciones dejan de estar en las tensiones con las que lidian, al intentar posicionarse y validarse como enseñantes ante los alumnos, y se trasladan a la interacción con los alumnos como una herramienta para conocerlos. Su principal preocupación radica en la necesidad de conocer a sus alumnos y en enfrentar la complejidad que presenta dicho proceso.

Un ámbito de preocupación que emerge a medida que van conociendo más a los alumnos, a través del trabajo tutorial, es cómo interactuar con alumnos y alumnas que denominan "complejos", cuyos nombres e historias serán traídos reiteradamente a la conversación hasta el final del taller. El conocimiento de las historias de vida de estos alumnos "complejos" será utilizado como marco explicativo para comprender su forma de actuar en el aula. En las conversaciones del taller empiezan aparecer miradas críticas respecto a cómo los profesionales de la escuela abordan las complejas situaciones de vida de estos alumnos. En el siguiente extracto se ejemplifica esta preocupación (sesión 6, semana 10 en aula):

\section{T1: ¿Quién es la Denisse?}

PFK: Una alumna que va a cumplir 14 años, está en $7^{\circ}$, y la niña, no, o sea la misma profesora (.) la de diferencial (...) me dijo que con Denisse ella había entregado la toalla, como que era un caso aparte, un comentario así. Yo con la Denisse, porque me tocó en mi grupo, he tenido como que hacer unos sistemas para que ella, por ejemplo no lee, ella no lee, tuve que convencer a los compañeros del curso para que le contaran el cuento a ella, porque no querían, porque es injusto según ellos que ellos lean y que la Denisse no lea, entonces ellos le tuvieron que contar el cuento y la Denisse por el momento ha participado pero siempre con la cara larga, siempre me manda a la punta del cerro $(\ldots)$

PFM: Hay que hablar con ella

PFC: Es que ella es una piedra

PFK: No, si ella me ha contado su vida, porque he tratado de hablar con ella, me ha contado su vida, ella me dice que anda pololeando, que primero vivía con la mamá pero que ahora se fue a vivir a RR, porque los papás son separados... Vive en RR pero dice que se cansa en la mañana porque tiene que venir caminando de para tomar la micro para venir para acá yo creo que también puede influir que llega cansada.... Me ha contado varias cosas de su vida, pero es cambiante, de repente a la otra clase llega muy, muy pesada luego como que se le pasa

I: Hay ciertos nombres de alumnos que han empezado a aparecer reiteradamente en la conversación, son niños y niñas con situaciones más complejas y creo que sería importante trabajar en cómo abordar esas situaciones 
M: es que el problema, es que son (...) son niñas con casos especiales, ahí tiene que haber una relación de cariño, tiene que haber una relación (...) y tú (se refiere a la profesora en formación) lo has logrado, porque te he visto trabajar con ella y que te escucha, cosas que con otras no ha hecho, y le cuesta mucho a ella poder trabajar con alguien por los problemas que ella tiene.

Como se evidencia en el extracto, la profesora en formación trae a la conversación su preocupación por una alumna particular, relatando su conocimiento de la historia de vida de la alumna y cómo utiliza esta información para explicar su comportamiento en el aula. Se aprecia también como este comportamiento conlleva ciertas dificultades para el trabajo en grupo. La practicante, además, emite un juicio crítico respecto de la falta de apoyo que la escuela ha dado a la alumna. La profesora en formación relata cómo ha ido buscando formas para enfrentar el desafío de trabajar con esta alumna en el aula. La intervención de la investigadora ayuda a la tríada a reflexionar sobre Denisse, como un ejemplo de uno de los desafíos de la docencia. La mentora le da retroalimentación positiva respecto al trabajo que ha realizado a través del cual ha logrado establecer un vínculo afectivo con la alumna.

Al transitar hacia la realización de la docencia colegiada al grupo curso, se producirá un segundo punto de inflexión. Sigue predominando como tema de conversación la interacción con los alumnos, pero la solicitud de una nueva demanda y el conocimiento adquirido de sus alumnos hará que sus preocupaciones se centren en cómo esas interacciones les permitirán impactar en el aprendizaje de sus alumnos.

En las últimas sesiones del taller emergerán nuevos tópicos de discursos. De los 32 tópicos de discurso que se presentaron en las últimas tres sesiones, 11 estuvieron centrados exclusivamente en el aprendizaje de sus alumnos. Los practicantes comenzaron a indagar y a reflexionar respecto a cómo mejorar el aprendizaje de sus alumnos, para lo cual buscaron, demandaron y utilizaron conocimientos de la disciplina, pedagogía, didáctica, psicología y evaluación. Sus preocupaciones se centraron en cómo conciliar el tener altas expectativas de sus alumnos, con ser capaces de diseñar tareas desafiantes pero posibles de ser abordadas, y que permitieran motivar y enriquecer el aprendizaje de sus alumnos. A continuación se presentan dos extractos (sesión 8 y 10) que evidencian la centralidad en el aprendizaje de alumnos y alumnas que caracteriza este último momento:

Extracto de la sesión 8 (semana 12 en aula):

\section{T1: PFC como lo harías tú en tu práctica a cargo del curso}

PFC: Igual es complejo, cuando nosotros hacemos las guías, de repente nosotros buscamos material que no fuese muy repetido, y yo pienso pucha será demasiado difícil, después digo a lo mejor no es tan difícil y yo creo que no se la van a poder, siempre estoy en conflicto con el tema de las guías.

T1: Pero, súper interesante lo que tú dices, porque otras experiencias educativas sostienen ah voy a poner todo en la guía, ni siquiera me pongo en el lugar del alumno en la empatía, podrán no podrán, independiente que lo que dice PFP también es muy cierto o sea mis expectativas tienen que tratar de elevar todos esos elementos, pese a lo cual yo debiera tener ese procedimiento de reflexión, podrán no podrán, así que bien, bien me parece. 
Estudios Pedagógicos XLII, N 4: 49-68, 2016

TRANSITANDO DESDE LA OBSERVACIÓN A LA ACCIÓN PEDAGÓGICA EN LA PRÁCTICA INICIAL: APRENDER A

ENSEÑAR CON FOCO EN EL APRENDIZAJE DEL ALUMNADO

PFC: De hecho lo que estaba tratando de hacer, es que hicimos el ítem 1 con los chiquillos (se refiere a los otros profesores en formación) y por ejemplo yo dije a lo mejor ellos no van a ser capaces de identificar por si solos los conectores, entonces les puse un cuadrito donde uno le da las opciones de conectores, obviamente ellos no tienen que ponerlo al achunte, pero sin embargo ellos tienen que identificar y poner en la oración, lo que nosotros le recalcamos que sea coherente, entonces ponerle solo el ejercicio yo pensaba que era demasiado y que no iban a entender y con el cuadrito sí iban a poder

Extracto de la sesión 10 (semana 17 en aula):

T2: Es que mi percepción era distinta del proceso que ustedes vivieron, ¿no hubo impacto?

PFP: No, es que yo creo que el impacto más grande fue hacia nosotros, nosotros lo sentimos más que los niños (el resto afirma síi), porque ellos a lo mejor están acostumbrados a tener practicantes, a lo mejor están acostumbrados a tal vez que van a estar por un tiempo y después se van a ir, porque incluso a mí me preguntaron "tío y ustedes hasta cuándo se van a quedar" (ríen), porque les extrañaba que siguiéramos tanto tiempo (todos ríen), pero nosotros como practicantes aprendimos yo creo que el triple o el cuádruple de los demás

PFM: Sí, yo creo que viene en nosotros el mayor aprendizaje, es que muchas mejoras uno no puede hacer porque estamos muy limitados por el tiempo, no se puede, a lo mejor los ayudamos en la clase del día a día, pero no sé si en su progreso general como estudiantes

PFP: Yo creo que en la clase, sí fue de bastante ayuda porque incluso muchos niños no se atreven a preguntarle a la profesora, pero sí a nosotros, tuvieron la confianza de levantar su mano y preguntarnos cada duda y nosotros siempre estábamos ahí respondiendo sus preguntas

PFT: Claro, porque por ejemplo Sergio (...) entonces igual me llamaba la atención que el solo llegara al lado mío a decirme sabe tía yo aprendí esto y esto otro, pucha entonces yo decía quizás es algo tan mínimo pero provocó un cambio en él

M: Se cumplió claramente el objetivo gracias al trabajo de todos, hubo un trabajo completamente dedicado a los alumnos, completamente enfocado en el buen desarrollo de las actividades.

I: Yo quisiera decir que el tema del impacto es bien subjetivo, hay veces que una experiencia acotada en el tiempo resulta lo suficientemente buena y profunda para lograr cambios significativos, y hay veces que experiencias muy largas no logran impacto, (...) entonces el clic que uno puede hacer, es que las acciones que uno hace no impactan solo por su cantidad sino también por su cualidad.

El extracto de la sesión 8 da cuenta del dilema que conlleva el diseño de material apropiado a las características de los estudiantes y cómo la profesora en formación debe discernir contemplando las expectativas y posibilidades de aprendizaje de sus alumnos, considerando cuál es la zona de desarrollo próximo en la que debería trabajar. El extracto de la sesión 10, evidencia cómo, ante la solicitud de los tutores respecto de reflexionar sobre lo que los practicantes han aprendido, en primera instancia, no respondan desde lo que 
ellos han aprendido como futuros docentes. Por el contrario, el interés de los practicantes radica en el aprendizaje de sus alumnos a partir del trabajo de ellos como enseñantes. Esta respuesta de los practicantes, da cuenta de su preocupación respecto a la evaluación que hacen de su práctica inicial en base al impacto de su trabajo en el aprendizaje de sus alumnos. Resulta interesante cómo estos profesores en formación van visualizando el valor de su quehacer para el aprendizaje de los alumnos, mostrando que si bien valoran el apoyo que brindaron en las clases, sus expectativas se encuentran en la búsqueda de un impacto mayor: el progreso educativo de sus alumnos.

\subsection{CONSTRUCCIÓN DE UNA AGENCIA PROFESIONAL EN PROFESORES EN FORMACIÓN}

A medida que los profesores en formación se vieron enfrentados a nuevas y más complejas tareas en el aula, se les fue demandando un rol más activo y profesional. Los resultados muestran cómo el aumento de complejidad de las tareas, desde ayudante de la docente a tutor de un grupo pequeño, a profesor del grupo curso, permitió otro aprendizaje profesional, la construcción de una agencia profesional. Los practicantes fueron tomando cada vez más iniciativas para elegir con cierto nivel de autonomía las acciones pedagógicas a implementar con sus estudiantes.

\subsubsection{Posicionamiento de profesores en formación: Transitando desde la observación hacia una posición agenciativa.}

Al integrarse a la práctica inicial, los practicantes sostuvieron una posición de espectadores. En las primeras sesiones del taller, más que conversaciones abundaron relatos con situaciones anecdóticas traídas por profesores en formación, y monólogos de consejería aportados por tutores y mentora. Como espectadores de una escena, iban relatando y comentando anécdotas, y luego iban recibiendo explicaciones o consejos de parte de expertos participantes, en este caso de tutores y mentora.

Durante el trabajo en tutorías a grupos pequeños, un nuevo conocimiento más personalizado y profundo de las historias de sus estudiantes les permitió asumir un posición opinante, utilizando el conocimiento construido para fundamentar sus explicaciones y razonamientos. Al trabajar en el diseño e implementación de una clase colegiada, los profesores en formación transitaron hacia una posición agenciativa. Dicha posición da cuenta de la construcción de un pensamiento crítico que les permitió cuestionar lo establecido, realizar razonamientos profesionales y tomar decisiones respecto al proceso de enseñanza-aprendizaje. Se observó cómo los practicantes abandonaron la búsqueda de respuestas únicas o recetas de expertos, para enfrentar situaciones complejas y empezar a construir un juicio profesional que asume que el trabajo docente se realiza en medio de dilemas que ameritan reflexión y trabajo colaborativo. El siguiente extracto (sesión 7, semana 12 en el aula) da cuenta de este nuevo posicionamiento:

PFM: Otra cosa, yo cuando hice la guía de puntuación, utilicé unas palabras que después expliqué abajo qué significaban, entonces la profesora nos dijo que nosotros no podíamos usar esas palabras, porque los niños no iban a entender. Entonces yo le dije por eso está la explicación abajo y yo le dije aparte si uno nunca usa palabras "complicadas", cómo espera que ellos siempre utilicen las mismas palabras. Entonces a ella misma le pasa que estamos leyendo 
Estudios Pedagógicos XLII, N 4: 49-68, 2016

TRANSITANDO DESDE LA OBSERVACIÓN A LA ACCIÓN PEDAGÓGICA EN LA PRÁCTICA INICIAL: APRENDER A

ENSEÑAR CON FOCO EN EL APRENDIZAJE DEL ALUMNADO

una poesía, que pregunten qué es travesía y son palabras que igual son comunes (...) hay que seguir reforzándolas para que ellos las usen como parte del vocabulario, no porque piense que ellos no me van a entender no voy a explayarme

PFK: Yo pienso también que uno tiene que saber eso si el significado de la palabra para poder explicárselas, porque si uno usa una palabra y no la sabe (ríe jejeje)

T1: Una palabra vigente

I: Y al final que primó, ¿la pusieron?

PFM: Sí, la pusimos y la PFT la explicó bien

T1: Por ejemplo, qué palabra, ¿te acuerdas?

PFM: Yuxtaponer

T2: Es fácil, es sencilla y la van a usar

PFK: Es una sana estrategia, o sea paulatinamente nadie dice mil quinientas palabras, pero paulatinamente deben ir incorporando vocabulario nuevo

El extracto ejemplifica cómo durante el diseño de la clase los practicantes van tomando decisiones por sí mismos y tomando caminos alternativos a los planteados por la mentora. Estas decisiones son justificadas desde una perspectiva profesional que contempla las capacidades que existen en sus alumnos y la relevancia de nuevos aprendizajes que amplíen sus conocimientos actuales. Al finalizar la práctica inicial en su cuarto semestre (de nueve que dura la carrera), la progresión en complejidad de las tareas realizadas, las nuevas demandas que deben enfrentar y la mediación en el taller ayudó a que estos futuros profesores lograran posicionarse como agentes en el proceso de aprendizaje de sus alumnos.

5.2.2 Aumento de expresiones agenciativas: críticas y acciones tomadas para asumir el quehacer profesional

Haapasaari et al. (2016) plantean seis expresiones agenciativas que se pueden presentar en los discursos: resistir el cambio, criticar la actividad o la organización actual, explicar nuevas posibilidades y potencialidades, idear nuevas formas o sugerencias orientadas hacia el futuro, comprometerse a tomar acciones concretas y reportar el haber realizado acciones concretas. Estas seis expresiones agenciativas aumentaron en su frecuencia a medida que transcurría el taller, siendo especialmente notorias en las últimas tres sesiones del taller (Véase gráfico $\mathrm{N}^{\circ} 1$ ). 
Gráfico 1. Evolución del número y tipo de expresiones agenciativas por sesión

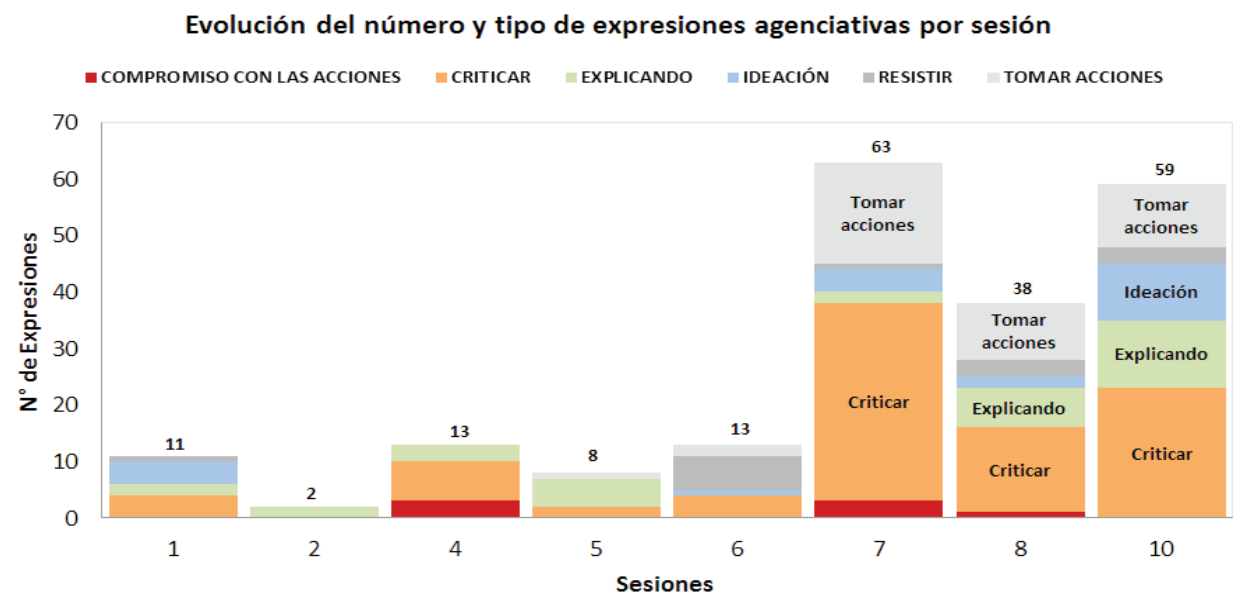

Fuente: elaboración propia

Tal como se aprecia en el gráfico, mientras los practicantes estuvieron abocados al diseño e implementación de las clases (sesiones 7, 8 y 10), aumentaron sus expresiones de críticas respecto a cómo el centro escolar abordaba las necesidades de aprendizaje de los estudiantes. También aumentaron las expresiones que aludían a que habían tomado acciones concretas para mejorar su trabajo. Por último, se observó un incremento en las expresiones de ideación y de explicación, situación que da cuenta cómo futuros profesores empiezan a imaginar nuevas posibilidades de ser profesor.

\subsection{SÍNTESIS DE RESULTADOS}

La Figura $\mathrm{N}^{\mathrm{o}} 1$ sintetiza la relación entre las modalidades de trabajo, los focos de preocupación de los practicantes y las distintas configuraciones del trabajo realizado por los tutores y la mentora en el taller de práctica. Los puntos de inflexión en la trayectoria de aprendizaje profesional de estos seis profesores en su práctica inicial se encontraron en la realización de tutorías y en la docencia colegiada al grupo curso. En la modalidad de ayudantía, los tutores y la mentora realizaban aportes complementarios a la preocupación de los practicantes. En la etapa de tutoría, a un grupo pequeño de estudiantes, se observó mayor coordinación entre los tutores universitarios y la mentora, junto a la incorporación de las voces de los estudiantes de las aulas escolares. En la modalidad docencia colegiada, el taller implicó un trabajo colaborativo en la mediación entre los tutores y la mentora, considerando la voz de los estudiantes. A través de estos cambios, los practicantes transitaron desde un foco en su enseñanza hacia un foco en sus estudiantes y su aprendizaje. Los estudiantes de pedagogía transitaron desde una posición de espectador, hacia opinantes, hacia una posición agenciativa para promover el aprendizaje en todos sus estudiantes. 
Figura 1. Trayectoria de aprendizaje profesional en práctica inicial

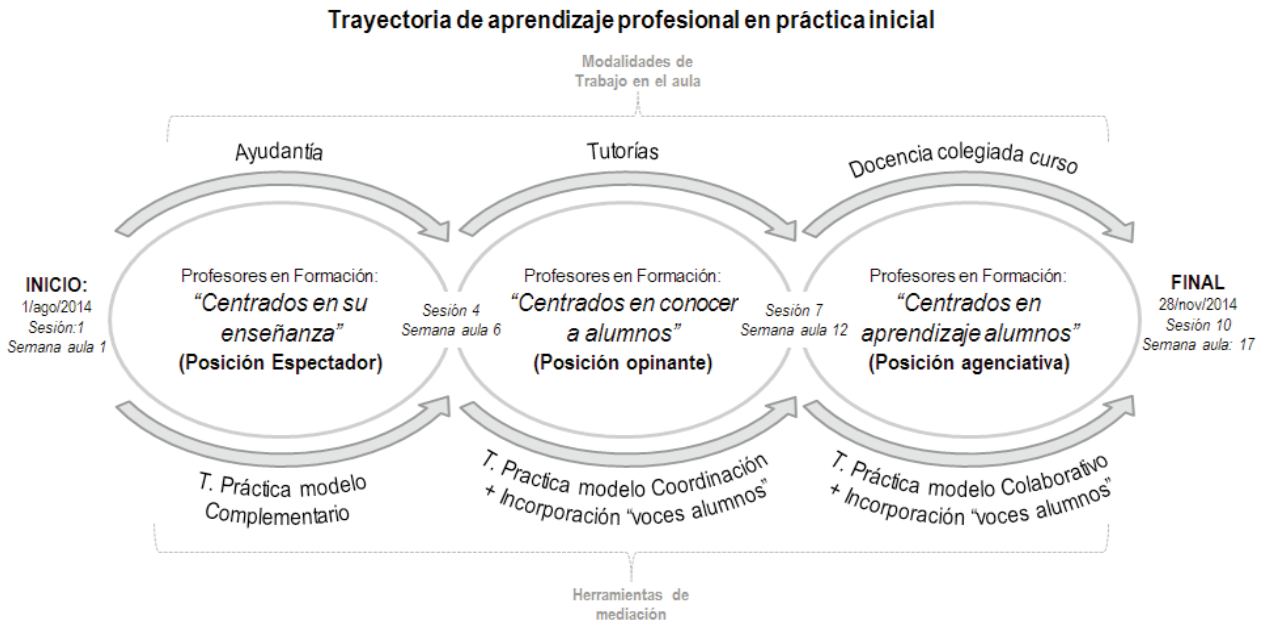

Fuente: elaboración propia

\section{DISCUSIÓN}

Este estudio ejemplifica una configuración (settings) para el aprendizaje de los futuros profesores situados en las aulas escolares. Esta configuración para la práctica inicial, diseñada de manera conjunta por la universidad y el centro escolar, enfrentó a los futuros profesores a tareas e interacciones que les permitieron acercarse a las auténticas demandas del trabajo docente. Las modalidades de trabajo que resultaron clave incluyeron la realización de tutorías a pequeños grupos y el diseño e implementación de clases colegiadas, que posibilitaron integrar conocimientos pedagógicos y disciplinares en función del conocimiento adquirido respecto a sus alumnos. En cuanto a las herramientas de mediación que resultaron fundamentales, encontramos el taller de práctica, que posibilitó una polifonía construida por la participación conjunta de tutores y mentora, y la incorporación de las voces de los estudiantes del sistema escolar.

Estas modalidades de trabajo y herramientas de mediación ofrecieron espacios de creación y colaboración que permitieron que la tríada formativa avanzara hacia la construcción de un objeto (motivo) compartido: el aprendizaje de los alumnos del centro escolar. La ayudantía inicial, al igual que la observación característica de la práctica inicial comúnmente implementada, tuvo poco impacto en propiciar aprendizaje profesional. Este bajo impacto se asocia al bajo nivel de organización y estructura de la actividad, ya que el objeto de aprendizaje era difuso (Brown, Collins \& Duguid, 1982). La ayudantía puede ser pertinente, pero por un período más breve (ejemplo, dos semanas) y con un objetivo claro (por ejemplo, conocer las rutinas de aula).

Involucrar a los futuros profesores en tareas auténticas del trabajo docente, en vez de tareas propias de un estudiante universitario, posibilitó que tempranamente en su proceso formativo pusieran mayor atención a cómo aprenden los alumnos, justamente lo que se 
espera de un docente (Korthagen et al., 2006; Desimone, 2009). Asumir el desafío de una formación práctica, que se proponga desde sus inicios el trasladar el núcleo de su quehacer desde la enseñanza hacia el aprendizaje de los alumnos, no es tarea sencilla. El estudio clásico de Fuller (1969) reporta dicho logro en profesores en formación recién durante la práctica final y solo a través de un adecuado acompañamiento.

El presente estudio da cuenta de la posibilidad de promover este foco en una práctica inicial diseñada en función de tareas auténticas respecto del desempeño docente, que aumentan en complejidad. Trabajar con un grupo de 5 a 6 alumnos por un periodo prolongado sin tener que planificar ese trabajo pedagógico permitió a los practicantes atender a cómo aprendían sus alumnos, a conocer a cabalidad sus características y necesidades de aprendizaje y a establecer relaciones de confianza con sus estudiantes. Con este bagaje de conocimientos, luego se embarcaron en la tarea de planificar clases con el apoyo de sus pares, la mentora y los tutores.

Habitualmente los talleres de práctica inicial se realizan en la universidad, en ausencia del profesor mentor, y no se solicita a los escolares entregar retroalimentación a los profesores practicantes (Solís et al., 2011). El presente estudio muestra los beneficios de que dicho taller posibilite la confluencia de experticias aportadas por distintos actores educativos que conforman la tríada formativa. La incorporación de la retroalimentación que entregan los alumnos del sistema escolar resultó una innovación generativa en el proceso formativo de los futuros profesores.

Los resultados de este estudio aportan a la actual discusión en Chile respecto del fortalecimiento de la formación práctica propuesta por la nueva ley de carrera docente (Ley 20.903), con mayores exigencias para la institucionalidad que apoya la formación práctica progresiva de los futuros profesores. Para que esta primera experiencia práctica se constituyera en un laboratorio para aprender a enseñar con foco en el aprendizaje de sus alumnos, se requirió de la asociación entre la universidad y el centro escolar. El tipo de colaboración evidenciado en la triada formativa requiere un apoyo institucional y no solo las voluntades individuales de sus miembros. La alianza institucional convocó, motivó, entregó orientaciones y condiciones para que la docente mentora y tutores fueran adaptando las tareas requeridas en función del foco de esta práctica inicial: que los practicantes desarrollaran una comprensión profunda respecto a cómo aprenden los estudiantes. El presente estudio señala que fortalecer la institucionalidad es importante pero requiere, además, atención a la elección de modelos teóricos que subyacen al diseño de la formación práctica.

Siguiendo un enfoque sociocultural, la progresión entre una etapa de práctica a la siguiente contempla variaciones en la complejidad de las tareas propias del trabajo docente, en las que confluyen las necesidades de aprendizaje de los estudiantes, de los centros escolares, como de los profesores en formación. Desde la etapa inicial, la formación práctica buscó generar oportunidades para aprender a pensar y actuar como profesor (Hammerness et al., 2005). Este diseño implicó al practicante en el desempeño de tareas auténticas y comprensivas que les permitieron interactuar y conocer a sus alumnos e ir analizando cómo avanzan y qué apoyos requieren para progresar en su aprendizaje. En esta línea, la propuesta de innovación para el componente de prácticas progresivas de los currículos de las carreras de pedagogía es un modelo que, a imagen de un fractal, pueda contenerse a sí misma y a su vez sea parte de la siguiente práctica. La apuesta es que su carácter progresivo sea dado por la amplitud y profundidad de las tareas, y por apoyos diferenciales que a 
modo de andamios puedan ir quitándose a medida que se transita de una práctica a otra. De ahí la relevancia de una cuidadosa articulación entre prácticas, que contemple un modelo sistémico y sinérgico entre ellas, conservando el carácter holístico que amerita la compleja tarea de formar a un futuro profesor. Este tipo de diseño contrasta con lo reportado por Contreras et al. (2010), en su análisis de currículos de formación práctica secuenciados en base a contenidos.

La construcción de un discurso pedagógico, cuyo centro es el aprendizaje del alumno del sistema escolar, posibilitó en estos seis profesores en formación una nueva mirada respecto a su propia trayectoria formativa. Poner como centro de atención el aprendizaje de alumnos y alumnas permitió a los profesores en formación aprender a reconocerlos y valorarlos en su diversidad, construyendo un sentido primario de equidad y justicia, impregnando así la acción educativa de un sentido ético enraizado en expandir las oportunidades para el desarrollo de las personas (Zeichner, 1996). Este sentido ético que reconoce el potencial de cada estudiante puede actuar como el corazón del proceso de aprender a enseñar, fortaleciendo el desarrollo de la identidad profesional. Si logramos que esta primera experiencia pedagógica tenga la fuerza para trazar un primer sendero cargado de sentido y propósito, de aquello que es primario y fundamental, es probable que se pueda facilitar que ese futuro profesor o profesora, en medio del cansancio o de las exigencias y demandas, rememore de vez en cuando ese primer sendero. Desde esa memoria podrá recobrar la vitalidad y el sentido de por qué y para qué eligió esta profesión.

\section{REFERENCIAS BIBLIOGRÁFICAS}

Ávalos, B. (2002). Profesores para Chile, historia de un proyecto. Santiago: MINEDUC. Recuperado el 7 de diciembre de 2014 desde http://biblioteca.uahurtado.cl/UJAH/Reduc/pdf/pdf/9161.pdf

Blanton, M., Berenson, S. \& Norwood, K. (2001). Exploring a pedagogy for educative supervision of prospective teachers. Journal of Mathematics Teacher Education, vol.4 (3), 177-204.

Borko, H. (2004). Professional development and teacher learning: mapping the terrain. Educational Researcher, vol.33 (8), 3-15.

Brown, J.S., Collins, A. \& Duguid, P. (1989). Situated cognition and the culture of learning. Educational Researcher, vol.18 (1), 32-42.

Brown, G. \& Yule, G. (1983). Discourse analysis. Cambridge: Cambridge University Press.

Clift, R.T. \& Brady, P. (2005). Research on methods courses and field experiences. In M. CochranSmith \& K. Zeichner (Eds.), Studying teacher education: the report of the AERA panel on research and teacher education (pp. 309-424). Mahwah: Lawrence Erlbaum Associates.

Comisión sobre Formación Inicial de Docentes. (2005). Informe Comisión sobre Formación Inicial Docente. Santiago, Chile.

Contreras, I., Rittershaussen, S., Montecinos, C., Solís, M., Núñez, C. y Walker, H. (2010). La escuela como espacio para aprender a enseñar: visiones desde los programas de formación de profesores de educación media. Estudios Pedagógicos, vol.36 (1), 85-105.

Correa Molina, E. (2014). Las prácticas: primer espacio de profesionalización docente. En I. Cortés y C. Hirmas (Eds.), Primer seminario sobre formación práctica docente: Vinculación entre el sistema universitario y el sistema escolar (pp. 25-40). Santiago: OEI

Darling-Hammond, L. (2013). Educar con calidad y equidad: los dilemas del siglo XXI. Revista de Investigación en Educación, vol.11 (1), 171-173. Recuperado el 5 de diciembre del 2015 desde http://webs.uvigo.es/reined/

. (1994). Developing professional development schools: early lessons, challenge and promise. 
In L. Darling-Hammond (Ed.), Professional development schools: Schools for developing a profession (pp.1-27). New York: Teachers College Press.

Desimone, L. (2009). Improving impact studies of teachers' professional development: toward better conceptualizations and measures. Educational Researcher, vol.38 (3), 181-199.

Donnellon, A. (1996). Team talk. The power of language in team dynamics. Boston: Harvard Business School Press.

Edwards, A. (2011). Building common knowledge at the boundaries between professional practices: relational agency and relational expertise in systems of distributed expertise. International Journal of Educational Research, vol.50 (1), 33-39.

Edwards, A. \& Mutton, T. (2007). Looking forward: rethinking professional learning through partnership arrangements in initial teacher education. Oxford Review of Education, vol.33 (4), 503-519.

Edwards, A. \& Protheroe, L. (2003). Learning to see in classrooms: what are student teachers learning about teaching and learning while learning to teach in schools? British Educational Research Journal, vol.29 (2), 227-242.

Ellis, V. (2010). Studying the process of change. In: V. Ellis \& A. Edwards (Eds.), Cultural-historical perspectives on teacher education and development: learning teaching (pp. 95-114). London: Routledge.

Engeström, Y. (2005). Developmental work research: expanding activity theory in practice. Berlin: International Cultural Historical Human Sciences.

Eri, T. (2013). The best way to conduct intervention research: methodological considerations. Quality \& Quantity, vol.47 (5), 2459-2472.

Fuller, F. (1969). Concerns of teachers: a developmental conceptualization. American Educational Research Journal, vol.6 (2), 207-226.

Greeno, J. (1998). The situativity of knowing, learning and research. American Psychologist, vol.53 (1), 5-26.

Haapasaari, A., Engeström, Y. \& Kerosuo, H. (2016). The emergence of learners' transformative agency in a change laboratory intervention. Journal of Education and Work, vol.29 (2), 232-262.

Hammerness, K., Darling-Hammond, L., Bransford, J., Berliner, D., Cochran-Smith, M., McDonald, M. \& Zeichner, K. (2005). How teachers learn and develop. In L. Darling-Hammond y J. Bransford (Eds.), Preparing teachers for a changing world: what teachers should learn and be able to do (pp. 358-389). Indianapolis: Jossey-Bass.

Hedegaard, M. (2012). The dynamic aspects in children's learning and development. In M. Hedegaard, A. Edwards \& M. Fleer, (Eds.), Motives, emotions and values in the development of children and young people (pp. 9-27). Cambridge: Cambridge University Press.

Korthagen, F. A., Loughran, J. \& Russell, T. (2006). Developing fundamental principles for teacher education programs and practices. Teaching and Teacher Education, vol.22 (8), 1020-1041.

Ludvigsen, S., Lund, A., Rasmussen, I. \& Säljö, R. (2011). Learning across sites: new tools, infrastructures and practices. In: S. Ludvigsen, A. Lund, I. Rasmussen \& R. Säljö (Eds.), Learning Across Sites (pp.1-13). Oxon: Routledge

Lunenberg, M. \& Korthagen, F. (2009). Experience, theory, and practical wisdom in teaching and teacher education. Teachers and Teaching, vol.15 (2), 225-240.

Méndez Zaballos, L. (2012). El conocimiento situado y los sistemas de actividad. Un modelo teórico para repensar el prácticum. Revista de Educación, (359), 359-155.

Mercer, N. (2004). Sociocultural discourse analysis: analysing classroom talk as a social mode of thinking. Journal of Applied Linguistics, vol.1 (2), 137-168.

Montecinos, C., Walker H., \& Maldonado, F. (2015). School administrators and university practicum supervisors as boundary brokers for initial teacher education in Chile. Teaching and Teacher Education, vol.49, 1-10. 
Montecinos, C., Cortez, M. \& Walker, H. (2015). School administrators' understandings and management of barriers for the school's involvement in the practicum component of initial teacher education in Chile. International Journal of Educational Development, vol.43, 100-108.

Montecinos, C., Walker, H., Rittershaussen, S., Nuñez, C., Contreras, I. \& Solís, M.C. (2011). Defining content for field-based coursework: contrasting the perspectives of secondary preservice teachers and their teacher preparation curricula. Teaching and Teacher Education, vol.27(2), 278-288.

Musset, P. (2010). Initial teacher education and continuing training policies in a comparative perspective: current practices in OECD countries and a literature review on potential effects. OECD Education Working Papers, 48, OECD Publishing. Recuperado el 22 de diciembre del 2014 desde http://dx.doi.org/10.1787/5kmbphh7s47h-en

Orland-Barak, L. (2005): Lost in translation: mentors learning to participate in competing discourses of practice. Journal of Teacher Education, vol.56 (4), 355-367.

Ortúzar, Ma. S., Flores, C. Milesi, C. \& Cox, C. (2009). Aspectos de la formación inicial docente y su influencia en el rendimiento académico de los alumnos. En I. Irarrázaval, E. Puga y M. Letelier (Eds.), Camino al Bicentenario. Propuestas para Chile. Santiago: PUC-Concurso de Políticas Públicas 2009. Recuperado el 5 de diciembre de 2013 desde http://www. sociedadpoliticaspublicas.cl/archivos/MODULO_II/Panel05_Educacion/Soledad_Ortuzar_ Aspectos_de_la_formacion_inicial_docente_y_su_influencia_en_el_rendimiento_acad.pdf

Panel de Expertos para una Educación de Calidad (2010). Propuestas para fortalecer la profesión docente en el sistema escolar chileno. Santiago: Ministerio de Educación.

Putnam, R.T. \& Borko, H. (2000). What do new views of knowledge and thinking have to say about research on teacher learning? Educational Researcher, vol.29 (1), 4-15.

Serrano, G.P. (1998). Investigación cualitativa. Retos e interrogantes. Madrid: La Muralla S.A.

Solís, M.C., Núñez, C., Contreras, I., Rittershaussen, S., Montecinos, C., \& Walker, H. (2011). Condiciones de la formación práctica de los futuros profesores. Estudios Pedagógicos, vol.37(1), 127-147.

Wenger, E. (2000). Communities of practice and social learning systems. Organization, vol.7(2), 225-246.

Zeichner, K. (2010). Rethinking the connections between campus courses and field experiences in college and university- based teacher education. Journal of Teacher Education, vol.61 (1-2), 89-99.

(1996). Teachers as reflective practitioners and the democratization of school reform. In

K. Zeichner, S., Melnick \& M. L., Gomez (Eds.), Currents of Reform in Preservice Teacher Education. New York: Columbia Teachers College.

Zeichner, K., Payne, K. \& Brayko, K. (2012). Democratizing knowledge in university teacher education through practice-based methods teaching and mediated field experience in schools and communities. Recuperado el 20 de enero 2014 desde http://www.ccte.org/wp-content/pdfsconferences/ccte-conf-2012-fall-zeichner-democratizing-knowledge.pdf. 